\title{
Determining the range of allowable axial force for the third-order Beam Constraint Model
}

\author{
Fulei Ma ${ }^{1}$, Guimin $\mathrm{Chen}^{1}$, and Guangbo $\mathrm{Hao}^{2}$ \\ ${ }^{1}$ School of Electro-Mechanical Engineering, Xidian University, Xi' an, Shaanxi 710071, China \\ ${ }^{2}$ School of Engineering-Electrical and Electronic Engineering, University College Cork, Ireland
}

Correspondence: Guimin Chen (guimin.chen@gmail.com)

Received: 20 July 2017 - Revised: 22 January 2018 - Accepted: 2 February 2018 - Published: 16 February 2018

\begin{abstract}
The Beam Constraint Model (BCM) was developed for the purpose of accurately and analytically modeling nonlinear behaviors of a planar beam flexure over an intermediate range of transverse deflections (10\% of the beam length). The BCM is expressed in the form of Taylor's expansion associated with the axial force. It has been found that the BCM may yield large predicting errors $(>5 \%)$ when the applied axial force goes beyond a certain boundary, even the deflection is still in the intermediate range. However, this boundary has not been clearly identified so far. In this work, we mathematically determine the non-dimensional boundary of the axial force by the condition that the strain energy expression of the BCM is a positive definite quadratic form, and by the buckling condition relate to compressing axial force. Several examples are analyzed to demonstrate the effects of the axial force on the modeling errors of the BCM. When using the BCM for modeling, it is always suggested to check if the axial force is within this boundary to avoid large modeling errors. If the axial force is beyond the boundary, the Chained Beam Constraint Model (CBCM) can be used instead.
\end{abstract}

\section{Introduction}

The Beam Constraint Model (BCM), developed by Awtar et al. (2007) a decade ago, offers a parametric and closed-form model for accurately and analytically capturing nonlinear behaviors of a planar beam flexure over an intermediate range of transverse deflections (typically when transverse motion is less than $10 \%$ of the beam length). The BCM is represented in the form of Taylor's expansion associated with the axial force. The commonly-used BCM keeps the first-order terms of the axial force (Awtar et al., 2007), termed as the firstorder BCM. The study objective of using the BCM is the slender beam (Euler beam) where beam length is 10 times longer than beam thickness in the bending direction. Due to its simplicity and analytical form, the BCM has been successfully used in characterizing the performances of tilted-beam compliant mechanisms (Awtar et al., 2007; Awtar and Sen, 2010a), $X Y$ flexure mechanisms (Awtar and Slocum, 2007), cross-spring flexural pivots (Zhao et al., 2011), fully compliant bistable mechanisms (Chen and Ma, 2015; Masters and Howell, 2003; Wilcox and Howell, 2005), and compound multibeam parallelogram mechanisms (Hao, 2015; Hao and
$\mathrm{Li}, 2015)$. The BCM can also capture the first fixed-free beam buckling mode where the buckling load $p_{\text {crit }}=-2.5$, which is less than $1.3 \%$ deviation from the classical beam buckling prediction of $p_{\text {crit }}=-\pi^{2} / 4$.

It was demonstrated that the maximum error of the first-order BCM is less than $5 \%$ for the non-dimensional transverse displacements (i.e., rotation $\theta$ and translation $y=\Delta Y / L$, as illustrated in Fig. 1) within \pm 0.1 , intermediate deflection range, and the normalized axial force $\left(p=P L^{2} /(E I)\right)$ within \pm 10 (Awtar et al., 2007). The non-dimensional transverse displacements are confined within \pm 0.1 because BCM was derived based on the linearized beam-curvature assumptions (Awtar et al., 2007). Moreover, the range of the normalized axial force $p$ was "empirically" restricted to $[-10,10]$ to maintain less than $2 \%$ truncation error (Awtar et al., 2010), which may limit the application of the BCM.

For compliant mechanisms that use flexible elements experiencing axial-force dominant loads, compressive force (Holst et al., 2011; Chen and Ma, 2015) or tensile force (Chen et al., 2009; Hao, 2015), there is a necessity 
to check if the maximum axial forces carried by the flexible elements are within the BCM's capability before using it for modeling. Moeen researched the nonlinear static load-displacement relationships of beam-based flexure modules with an intermediate semi-rigid element (Moeen and Moeenfard, 2017) and considered the load-stiffening effects caused by axial loads using the BCM (Moeen and Moeenfard, 2018). Hao (2015) showed that the results of the firstorder BCM for compound multi-beam parallelogram mechanisms significantly deviate from the results of the BCM including third-order terms of the axial force (termed as the third-order $\mathrm{BCM}$ ) when the axial force exceeds a certain value. Although the third-order BCM is more accurate than the first-order $\mathrm{BCM}$ and extends the use of the $\mathrm{BCM}$, what is the allowable axial force range of the third-order BCM remains unknown.

The determination of the range of allowable axial force includes finding the lower axial force boundary $p_{1}$ (i.e. the maximum compressive axial force) and the upper boundary $p_{\mathrm{u}}$ (i.e. the maximum tensile axial force). Therefore, in this paper, by using the conditions: (1) the positive definite quadratic condition of the strain energy expression of the $\mathrm{BCM}$; (2) the characteristic that the BCM can only capture the first buckling mode (the deflected beam carrying no more than one inflection point), we mathematically derive the upper and lower bounds of the allowable axial force for the third-order BCM, corresponding to the maximum tensile and compressive forces that the BCM can take, respectively. The third-order model is considered in this work because Hao (2015) showed that the BCM with the third-order terms accurately captures the relevant nonlinearities.

The rest of this paper is organized as follows. Section 2 briefly reviews the $\mathrm{BCM}$ equations and other related wor. The range of allowable axial force for BCM is mathematically obtained in Sects. 3.1 and 4.1. Three examples are utilised in Sects. 3.2 and 4.2 to demonstrate how the axial force influences the modeling errors of the third BCM. Finally, Sect. 5 draws remarks of this study.

\section{Literature review}

\subsection{Revisiting the Beam Constraint Model (BCM)}

Figure 1 illustrates a uniform cross-section beam flexure imposed by a transverse force $F$, an axial force $P$ and a moment $M$ at its free end, resulting in transverse and axial deflections $\Delta X, \Delta Y$ and a tip slope $\theta$. The parameters of the beam include: the length $L$, the in-plane thickness $T$, the out-ofplane thickness $H$, and the Young's modulus of the material $E$. $A=H T$ and $I=H T^{3} / 12$ are the area and the second moment of inertia of the beam's cross-section, respectively. The BCM formulates the nonlinear load-deflection relations of the beam using a set of parametric and closed-form equations (Awtar et al., 2010). Following are the BCM equations with the third-order terms of the axial force included (Wilcox

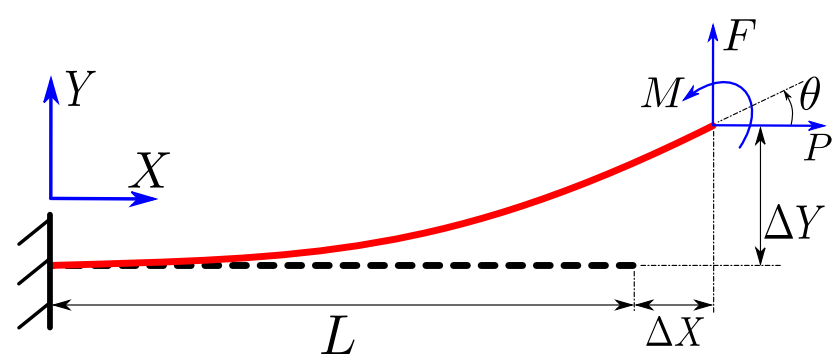

Figure 1. A simple beam flexure subject to combined force and moment loads.

and Howell, 2005; Hao et al., 2011):

$$
\begin{aligned}
& \left(\begin{array}{c}
f \\
m
\end{array}\right) 7=\left(\begin{array}{ll}
k_{11}^{(0)} & k_{12}^{(0)} \\
k_{12}^{(0)} & k_{22}^{(0)}
\end{array}\right)\left(\begin{array}{l}
y \\
\theta
\end{array}\right)+p\left(\begin{array}{ll}
k_{11}^{(1)} & k_{12}^{(1)} \\
k_{12}^{(1)} & k_{22}^{(1)}
\end{array}\right)\left(\begin{array}{l}
y \\
\theta
\end{array}\right) \\
& +p^{2}\left(\begin{array}{ll}
k_{11}^{(2)} & k_{12}^{(2)} \\
k_{12}^{(2)} & k_{22}^{(2)}
\end{array}\right)\left(\begin{array}{l}
y \\
\theta
\end{array}\right)+p^{3}\left(\begin{array}{ll}
k_{11}^{(3)} & k_{12}^{(3)} \\
k_{12}^{(3)} & k_{22}^{(3)}
\end{array}\right)\left(\begin{array}{l}
y \\
\theta
\end{array}\right) \\
& x=\frac{t^{2} p}{12 L^{2}}-\frac{1}{2}\left(\begin{array}{ll}
y & \theta
\end{array}\right)\left(\begin{array}{ll}
k_{11}^{(1)} & k_{12}^{(1)} \\
k_{12}^{(1)} & k_{22}^{(1)}
\end{array}\right)\left(\begin{array}{l}
y \\
\theta
\end{array}\right) \\
& -p\left(\begin{array}{ll}
y & \theta
\end{array}\right)\left(\begin{array}{ll}
k_{11}^{(2)} & k_{12}^{(2)} \\
k_{12}^{(2)} & k_{22}^{(2)}
\end{array}\right)\left(\begin{array}{l}
y \\
\theta
\end{array}\right) \\
& -\frac{3}{2} p^{2}\left(\begin{array}{ll}
y & \theta
\end{array}\right)\left(\begin{array}{ll}
k_{11}^{(3)} & k_{12}^{(3)} \\
k_{12}^{(3)} & k_{22}^{(3)}
\end{array}\right)\left(\begin{array}{l}
y \\
\theta
\end{array}\right) \\
& -p^{3}\left(\begin{array}{ll}
y & \theta
\end{array}\right)\left(\begin{array}{ll}
g_{11}^{(3)} & g_{12}^{(3)} \\
g_{12}^{(3)} & g_{22}^{(3)}
\end{array}\right)\left(\begin{array}{l}
y \\
\theta
\end{array}\right)
\end{aligned}
$$

where the non-dimensional coefficients $k$ 's and $g$ 's in the matrices are entirely independent of the beam shape and are referred to as the beam characteristic coefficients, as listed in Table 1. $t$ is the normalized thickness given as $t=T / L$. The variables, $m, f$ and $p$, are the normalized load parameters and $y$ and $x$ are the non-dimensionalized (normalized) deflection parameters, which are, respectively, given as:

$m=\frac{M L}{E I}, f=\frac{F L^{2}}{E I}, p=\frac{P L^{2}}{E I}, y=\frac{\Delta Y}{L}, x=\frac{\Delta X}{L}$

In addition, the third-order strain energy stored in the deflected beam is formulated as (Awtar et al., 2010; Awtar and Sen, 2010b)

$$
\begin{aligned}
v & =\frac{1}{2} \frac{t^{2} p^{2}}{12 L^{2}}+\frac{1}{2}\left(\begin{array}{ll}
y & \theta
\end{array}\right)\left(\begin{array}{ll}
k_{11}^{(0)} & k_{12}^{(0)} \\
k_{12}^{(0)} & k_{22}^{(0)}
\end{array}\right)\left(\begin{array}{l}
y \\
\theta
\end{array}\right) \\
& -\frac{1}{2} p^{2}\left(\begin{array}{ll}
y & \theta
\end{array}\right)\left(\begin{array}{ll}
k_{11}^{(2)} & k_{12}^{(2)} \\
k_{12}^{(2)} & k_{22}^{(2)}
\end{array}\right)\left(\begin{array}{l}
y \\
\theta
\end{array}\right) \\
& -p^{3}\left(\begin{array}{ll}
y & \theta
\end{array}\right)\left(\begin{array}{ll}
k_{11}^{(3)} & k_{12}^{(3)} \\
k_{12}^{(3)} & k_{22}^{(3)}
\end{array}\right)\left(\begin{array}{l}
y \\
\theta
\end{array}\right)
\end{aligned}
$$


Table 1. Beam characteristic coefficients of the BCM matrices (Awtar et al., 2010).

\begin{tabular}{rrrrrr}
\hline$k_{11}^{(0)}$ & 12 & $k_{12}^{(0)}$ & -6 & $k_{22}^{(0)}$ & 4 \\
$k_{11}^{(1)}$ & $6 / 5$ & $k_{12}^{(1)}$ & $-1 / 10$ & $k_{22}^{(1)}$ & $2 / 15$ \\
$k_{11}^{(2)}$ & $-1 / 700$ & $k_{12}^{(2)}$ & $1 / 1400$ & $k_{22}^{(2)}$ & $-11 / 6300$ \\
$k_{11}^{(3)}$ & $1 / 63000$ & $k_{12}^{(3)}$ & $-1 / 126000$ & $k_{22}^{(3)}$ & $1 / 27000$ \\
$g_{11}^{(3)}$ & $-37 / 97020000$ & $g_{12}^{(3)}$ & $37 / 194040000$ & $g_{22}^{(3)}$ & $-509 / 291060000$ \\
\hline
\end{tabular}

in which $v$ is the normalized strain energy with respect to the beam parameters given as

$v=\frac{V L}{E I}$

where $V$ is the actual strain energy.

Note that the values of coefficients in Eqs. (1)-(4) are listed in Table 1.

\subsection{Other related work}

Hao et al. (2011) proposed an analytical method for modeling spatial deflection of a slender beam in its intermediate deflection range, which combines two orthogonal beam constraint models (BCMs) with a torsional deflection model in the deformed configuration. Based on the Timoshenko beam theory, the Timoshenko Beam Constraint Model (TBCM) was developed for the purpose of including shear effects in the model (Chen and Ma, 2015). Sen and Awtar (2013), Sen (2013) presented a spatial BCM (SBCM) for the purpose of accurately predicting the nonlinear spatial constraint characteristics of thin bisymmetric beams in their intermediate deflection range (a bisymmetric beam refers to a beam whose cross section has equal moments of area and zero product of inertia). Ma and Chen (2016) proposed a Chained BCM (CBCM), which extends the BCM for modeling large planer deflections of beams in compliant mechanisms by utilizing a discretization strategy. The CBCM also alleviates the limitation of the maximum axial load of the BCM through discretization. Chen and Bai (2015) further developed a Chained SBCM (CSBCM), which extends the SBCM for modeling large spatial deflections of bisymmetric flexible beams.

\section{Upper bound of axial force}

\subsection{Determining upper bound}

The strain energy stored in the deflected beam (Eq. 4) can be rewritten as:

$v=\frac{V L}{E I}=\frac{1}{2} \frac{t^{2} p^{2}}{12 L^{2}}+\frac{1}{2}\left[\begin{array}{ll}y & \theta\end{array}\right]\left[\begin{array}{ll}K_{\mathrm{e} 11}(p) & K_{\mathrm{e} 12}(p) \\ K_{\mathrm{e} 21}(p) & K_{\mathrm{e} 22}(p)\end{array}\right]\left[\begin{array}{l}y \\ \theta\end{array}\right]$

where the first term on the right hand side of Eq. (5) represents the strain energy due to stretching or compressing and the following second term represents the strain energy due to bending. The $K_{\mathrm{e}}(p)$ 's in the strain energy formulation are

$$
\begin{aligned}
& K_{\mathrm{e} 11}(p)=k_{11}^{(0)}-k_{11}^{(2)} p^{2}-2 k_{11}^{(3)} p^{3} \\
& K_{\mathrm{e} 12}(p)=k_{12}^{(0)}-k_{12}^{(2)} p^{2}-2 k_{12}^{(3)} p^{3} \\
& K_{\mathrm{e} 21}(p)=k_{12}^{(0)}-k_{12}^{(2)} p^{2}-2 k_{12}^{(3)} p^{3} \\
& K_{\mathrm{e} 22}(p)=k_{22}^{(0)}-k_{22}^{(2)} p^{2}-2 k_{22}^{(3)} p^{3}
\end{aligned}
$$

Because the strain energy expression of the third-order BCM is in a positive definite quadratic form for the case with a tensile axial force. The following conditions should be satisfied (Gilbert, 2009):

$$
\begin{aligned}
& K_{\mathrm{e} 11}(p)>0 \\
& K_{\mathrm{e} 11}(p) K_{\mathrm{e} 22}(p)-K_{\mathrm{e} 12}(p) K_{\mathrm{e} 21}(p)>0
\end{aligned}
$$

Combining Eqs. (6) and (7) and substituting the beam characteristic coefficients in Table 1 into the result yields the following inequalities:

$$
\begin{aligned}
& -\frac{p^{3}}{31500}+\frac{p^{2}}{700}+12>0 \\
& \frac{p^{6}}{476280000}-\frac{11 p^{5}}{79380000}+\frac{p^{4}}{504000}-\frac{13 p^{3}}{15750} \\
& \quad+\frac{19 p^{2}}{1050}+12>0
\end{aligned}
$$

By solving the above inequalities, we have:

$$
p<34.05
$$

That is to say, $p_{\mathrm{u}}=34.05$ is the upper bound of axial force when using the third-order BCM. It should also be noted that a Maximum truncation error of $2.4 \%$ is occurred with the neglecting of the item with the fourth power of $p$ in the polynomial.

\subsection{Case study}

A compliant parallelogram mechanism, as shown in Fig. 2, is widely used in precision positioning as compliant translational joints (Howell, 2001). The geometric nonlinearities associated with deflections of the flexible beams and the resulting nonlinear behaviors always complicate the modeling. For example, when applying a vertical force $F_{y}$ at the motion 


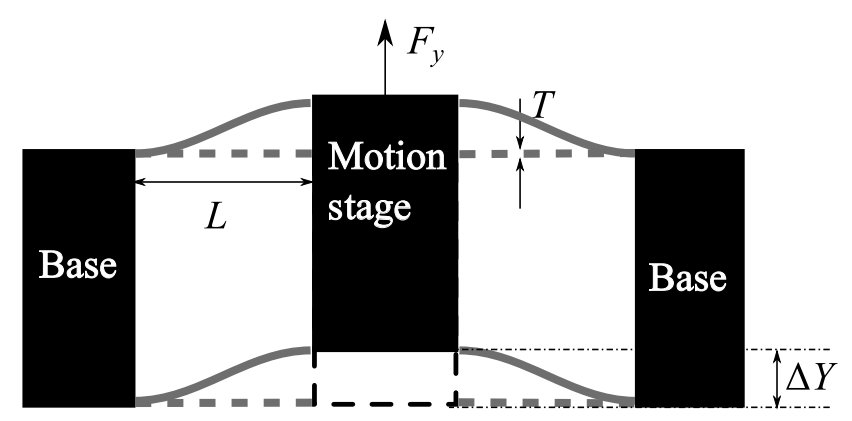

Figure 2. A fixed-guided beam in compliant parallelogram mechanism.

Table 2. Geometric parameters of the compliant parallelogram mechanism. $H$ is the out-of-plane thickness of the beam.

\begin{tabular}{lllll}
\hline Parameter & $H$ & $L$ & $T$ & $E$ \\
\hline Value & $20 \mathrm{~mm}$ & $45 \mathrm{~mm}$ & $1 \mathrm{~mm}$ & $69 \mathrm{GPa}$ \\
\hline
\end{tabular}

stage of the compliant parallelogram mechanism, the internal tensile axial force along the flexible beam can become very large while the transverse displacement $\Delta Y$ restrict to within $10 \%$ of the beam length. The BCM is a reasonable choice to model the flexible beams in compliant parallelogram mechanisms. Hao (2015) used the third-order BCM for modeling compound compliant parallelogram mechanisms. But when the transverse displacement $\Delta Y$ increases to a certain value, the difference between FEA and the BCM becomes large so that the third-order BCM may fail to predict the correct forcedisplacement relationship. The reason is that when the nondimensional axial force $p$ exceeds the upper boundary axial force $p_{\mathrm{u}}$ given in Eq. (9), the third-order BCM begins to predict the inaccurate results, in other words, the third-order BCM should be used within the allowable upper boundary of the axial force. More details are followed below.

Since the compliant parallelogram mechanism has a symmetric configuration, only a single beam of the mechanism is modeled. As shown in Fig. 3, the beam parameters listed in Table 2 are identical to those used in Hao (2015). The coordinate $X O Y$ is established with its origin at the fixed end attached to the base. The normalized end displacement can be written as:

$x=0, \quad y=\frac{\Delta Y}{L}, \quad \theta=0$

Let $\Delta Y$ increase from the initial position to $0.1 L$ and substitute Eq. (10) into Eqs. (1) and (2), the relationships of the reaction force $F_{y}$ and the normalized internal axial force $p$ along the beam against the transverse displacement $\Delta Y$ can be illustrated in Figs. 4 and 5, respectively.

A finite element model of the compliant parallelogram mechanism was built with the ANSYS software where the beam was meshed into 200 elements with Beam 188. The

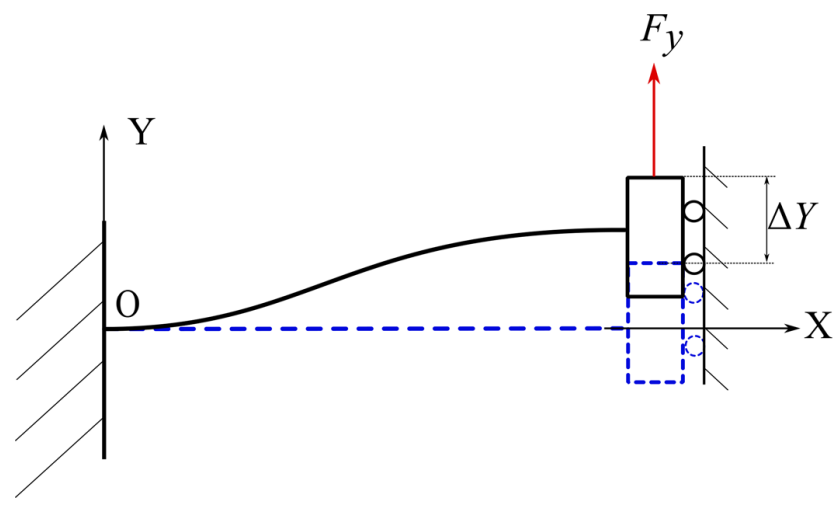

Figure 3. A beam flexure in compliant parallelogram mechanism.

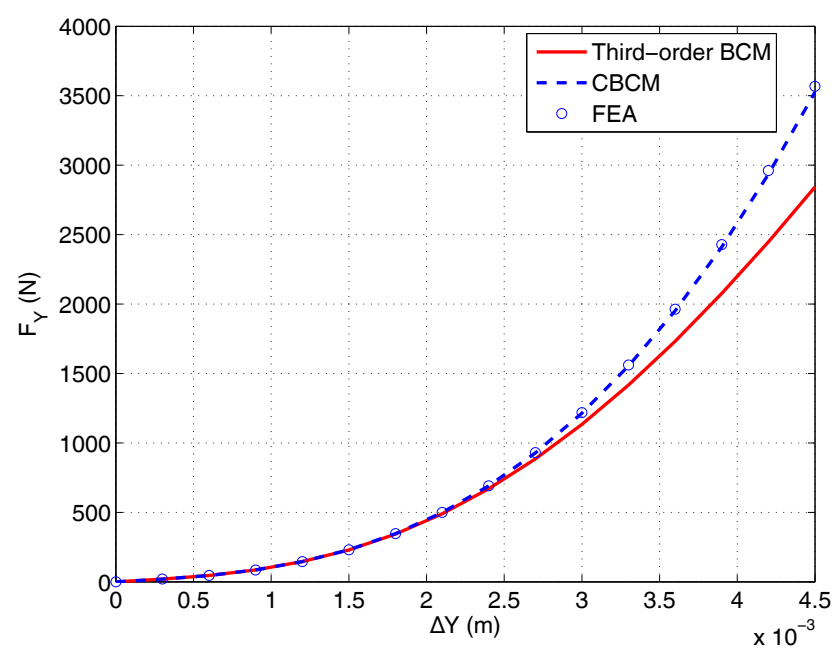

Figure 4. Comparion of the load-displacement relationship of the compliant parallelogram mechanism.

results are also plotted in Fig. 4. It can be seen from Fig. 4 that the results obtained by the third-order BCM clearly deviate from FEA results after $p$ exceeds $p_{\mathrm{u}}$. The maximum error of the BCM comparing with FEA is $20 \%$ when the non-dimensional end displacement is 0.1 . The axial tensile loads are also given in Fig. 5 which shows that the maximum internal axial tensile force of the third-order BCM is larger than 100 , which is much larger the upper boundary of the allowable force $p_{\mathrm{u}}$ for the BCM.

In order to show the effect of the axial tensile force on the modeling error of the third-order BCM, we compare its results to those obtained using CBCM. Considering the effectiveness and efficiency, the CBCM results were obtained by dividing the beam into 3 elements (Ma and Chen, 2016). In such a way, the element axial tensile loads by normalization is reduced to $p_{\mathrm{e}}=P(L / 3)^{2} / E I=p / 9$ which is ensured to be less than $p_{\mathrm{u}}$. The maximum error of the CBCM is reduced to $1 \%$, and the curves of the CBCM error and the normalized internal axial force during the deflection are also plotted in Fig. 5. Although not discussed in this work, it should be 


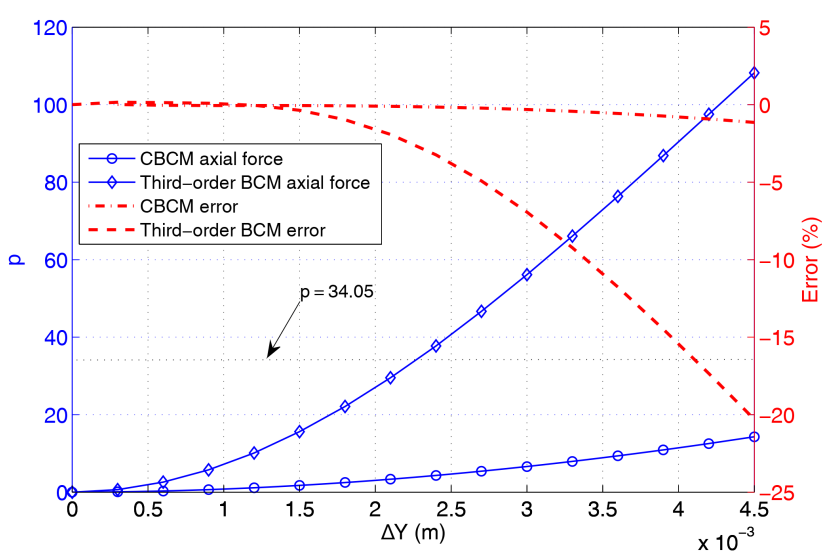

Figure 5. Comparision of normalised axial force and error of the compliant parallelogram mechanism.

noted that the maximum stress in the beams may exceed the yield stress of the material when the mechanism translates $0.1 \mathrm{~L}$ along the transverse direction, inducing failure of the mechanism.

\section{Lower bound of axial force}

\subsection{Determining lower bound}

When the compressive force applied at the end of a flexible beam exceeds a certain value $p$, the beam can buckle due to the instability of the beam. Actually, the buckling corresponds to a singularity of the stiffness matrix in the forcedisplacement formula. This buckling load is also defined as the critical load $f_{\text {cr. }}$. As the third-order BCM is derived based on a fixed-free beam shown in Fig. 1, the compressive force related to the buckling can be calculated by setting the determinant of the nonlinear bending stiffness matrix to be zero. Rewrite Eq. (1) as:

$$
\left(\begin{array}{c}
f \\
m
\end{array}\right)=\mathbf{K}_{\mathrm{s}}\left(\begin{array}{c}
y \\
\theta
\end{array}\right)=\left(\begin{array}{ll}
\mathbf{K}_{\mathrm{s} 11}(p) & \mathbf{K}_{\mathrm{s} 12}(p) \\
\mathbf{K}_{\mathrm{s} 21}(p) & \mathbf{K}_{\mathrm{s} 22}(p)
\end{array}\right)\left(\begin{array}{c}
y \\
\theta
\end{array}\right)
$$

where $\mathbf{K}_{\mathrm{s}}$ is the nonlinear stiffness matrix that relates the tip loads and the tip displacements, in which each

$$
\begin{aligned}
& \mathbf{K}_{\mathrm{s} 11}(p)=k_{11}^{(0)}+k_{11}^{(1)} p+k_{11}^{(2)} p^{2}+k_{11}^{(3)} p^{3} \\
& \mathbf{K}_{\mathrm{s} 12}(p)=k_{12}^{(0)}+k_{12}^{(1)} p+k_{12}^{(2)} p^{2}+k_{12}^{(3)} p^{3} \\
& \mathbf{K}_{\mathrm{s} 21}(p)=k_{12}^{(0)}+k_{12}^{(1)} p+k_{12}^{(2)} p^{2}+k_{12}^{(3)} p^{3} \\
& \mathbf{K}_{\mathrm{s} 22}(p)=k_{22}^{(0)}+k_{22}^{(1)} p+k_{22}^{(2)} p^{2}+k_{22}^{(3)} p^{3}
\end{aligned}
$$

By letting the determinant of $\mathbf{K}_{\mathrm{s}}$ be zero and using the parameters given in Table 1, the following equation can be ob- tained:

$$
\begin{gathered}
\frac{p^{6}}{1905120000}-\frac{11 p^{5}}{158760000}+\frac{71 p^{4}}{1512000}-\frac{109 p^{3}}{63000} \\
+\frac{277 p^{2}}{2100}+\frac{26 p}{5}+12=0
\end{gathered}
$$

Equation (13) is solved to obtain the following roots:

$$
p=\left\{\begin{array}{l}
47.16+83.74 i \\
47.16+83.74 i \\
31.82+61.26 i \\
31.82-61.26 i \\
-23.50 \\
-2.47
\end{array}\right.
$$

The first four roots are complex numbers with no physical meaning, which thus are neglected. The fifth and sixth roots are real solutions associated with two buckling modes. However, when the normalized compressive axial force increases to the lower one, $p=-2.47$, the stiffness matrix $\mathbf{K}_{\mathrm{s}}$ will exhibit a singularity, which the beam buckles, so the larger one (fifth root) is not considered. The axial force $f_{\mathrm{a}}$ corresponding to the first buckling mode of fixed-free beam is given as:

$p_{\mathrm{a}}=p=-2.47$

It is indicated in the knowledge of mechanics of materials (Timoshenko, 2001) that the critical load $f_{\text {cr }}$ corresponding to buckling is closely related to the boundary condition applying at the end of the flexible beam. In order to find the lower boundary of the axial force that defines the third-order BCM formula, we consider others boundary conditions: fixed-pivoted and fixed-fixed, as illustrated in Fig. 6. The critical loads corresponding to the fixed-free, fixedpivoted and fixed-fixed beam buckling modes (Fig. 6) can be written, respectively, as:

$$
\begin{aligned}
& p_{\mathrm{a}}=f_{\mathrm{cr}} \\
& p_{\mathrm{b}}=\frac{400}{49} f_{\mathrm{cr}} \\
& p_{\mathrm{c}}=16 f_{\mathrm{cr}}
\end{aligned}
$$

As the BCM can only capture the first buckling mode, according to the results in Eq. (16), the lower boundary of the axial force, $p_{1}$, for different boundary conditions can be expressed as:

$p_{1}= \begin{cases}-2.47 & \text { Fixed-free beam } \\ -20.16 & \text { Fixed-pivoted beam } \\ -39.52 & \text { Fixed-fixed beam }\end{cases}$

\subsection{Case study}

The bistable mechanism shown in Fig. 7 has many potential applications such as switches, static balancing mechanisms. 


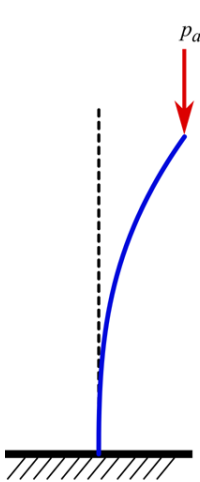

(a)

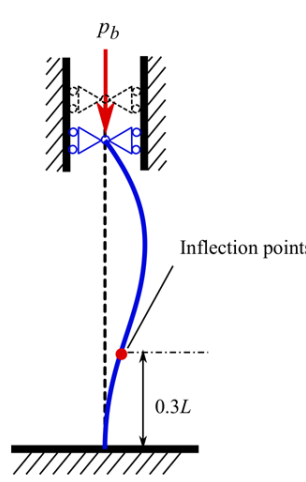

(b)

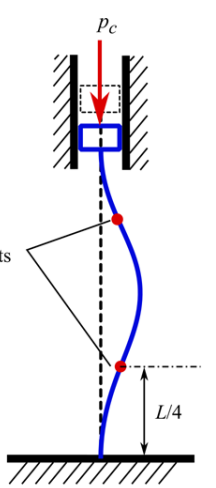

(c)
Figure 6. (a) fixed-free beam buckling; (b) fixed-pivot beam buckling; (c) fixed-fixed beam buckling.

The negative stiffness and the bistable behaviors have always complicated the modeling. Furthermore, the compressive axial force along the flexible beam can become very large while the transverse displacement $\Delta Y$ (perpendicular to the beam) is still limited within $10 \%$ of the beam length. Among all the methods, the BCM is a prior choice to model the behaviors of bistable mechanisms. But, when the compressive axial force exceeds a certain value, the BCM results may show an error. In this section, two different bistable mechanism examples were used to demonstrate the effectiveness of the range of the axial force allowable for the BCM. The first example is chosen to show the performance of the BCM when the compressive axial force is larger than the allowable lower boundary. The second example, on the contrary, is a case for the compressive axial force within the allowable lower boundary.

It should be demonstrated that for both examples the nondimensional transverse displacements perpendicular to the beam are strictly limited within \pm 0.1 in all the deflected cases in order to guarantee the effectiveness of the linearized approximate expression for curvature. Details are elaborated as follows.

Considering that the geometry and loading of the bistable mechanism are symmetrical, a single fixed-guided limb is chosen to analyze. Figure 8 illustrates the schematic of the fixed-guided beam. Establish the coordinate system along the beam with its origin placed at the fixed end and denote the initial angle of the fixed-guided beam as $\beta$. The end displacement can be written as:

$x=\frac{\Delta Y \cos \beta}{L}, \quad y=\frac{\Delta Y \sin \beta}{L}, \quad \theta=0$

Where $x$ is the normalized displacement along the beam, $y$ is the normalized displacement perpendicular to the beam, and $\theta$ is the tip rotation of the beam, which stays unchanged during the deflection. The parameters of Example I are given in Table 3. Let $\Delta Y$ increase from the initial position to $0.1 \mathrm{~L}$ with the help of submitting Eq. (18) into Eqs. (1) and (2),

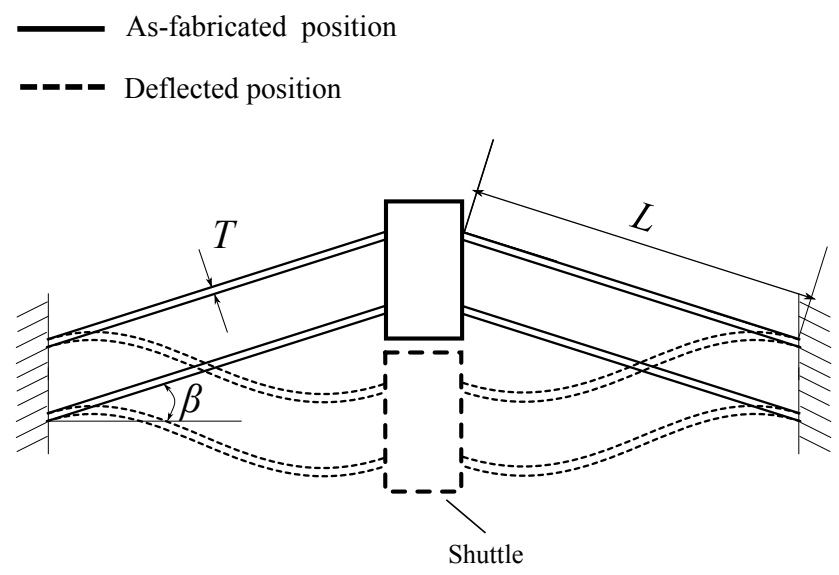

Figure 7. A bistable mechanism.

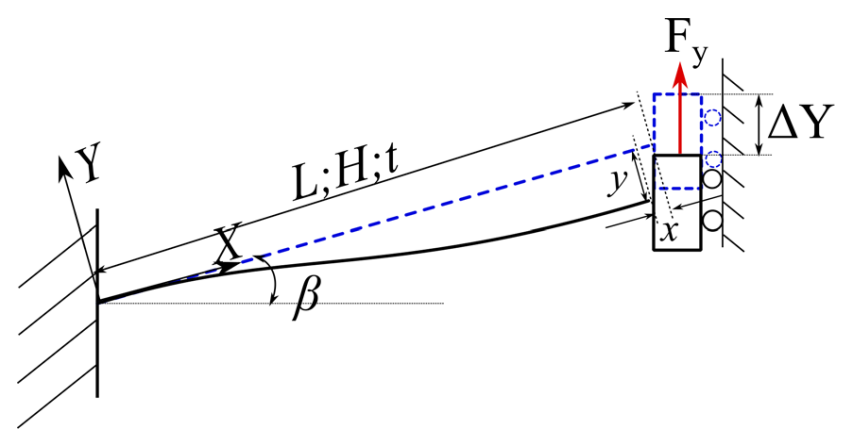

Figure 8. Schematic of the bistable mechanism.

the relationships of the reaction force $F_{y}$ and the normalized internal axial force along the beam against the transverse displacement $\Delta Y$ can be obtained as shown in Figs. 9 and 10, respectively.

Modelling the deflected beam of Example I using an FEA model built with the ANSYS software (the beam was meshed into 200 elements with Beam 188), the FEA forcedisplacement relationship is also plotted in Fig. 9. It can be observed that the results achieved by the BCM are consistent well with those obtained by FEA at the beginning of the force-deflection curve. When the tip displacement $\Delta Y$ getting larger, the third-order BCM fails to predict the forcedisplacement relationship. The error between the results obtained by the BCM and FEA is up to $60 \%$, as shown in Fig. 10. The normalized internal axial force along the beam during the deflection is also illustrated in Fig. 10 (using the other $y$ axis for comparison in the figure). It can be shown that when the normalized compressing axial force magnitude $p$ is larger than 39.52 , which exceeds the lower boundary of the axial force $p_{1}$, the third-order BCM fails to deal with this problem.

The problem in Example I can be easily solved and the axial force at each displacement step was obtained by numerically solving the CBCM equations (Ma and Chen, 2016), The 
Table 3. Parameters of the examples. $H$ is the out-of-plane thickness of the beam.

\begin{tabular}{lll}
\hline Parameter & Example I & Example II \\
\hline$H$ & $10 \mathrm{~mm}$ & $10 \mathrm{~mm}$ \\
$L$ & $80 \mathrm{~mm}$ & $60 \mathrm{~mm}$ \\
$\theta$ & $3.5^{\circ}$ & $2^{\circ}$ \\
$t$ & $1 \mathrm{~mm}$ & $1 \mathrm{~mm}$ \\
$E$ & $1.379 \mathrm{GPa}$ & $1.379 \mathrm{GPa}$ \\
\hline
\end{tabular}

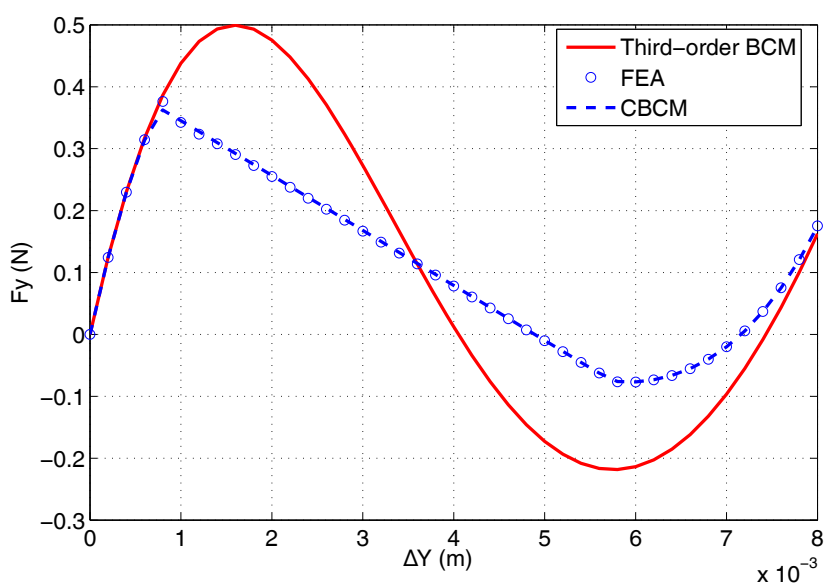

Figure 9. Comparison of the load-displacement relationship of Example I.

curve obtained by the CBCM is given in Fig. 9. The error of the force displacement relationship obtained by comparing the CBCM and FEA results is also plotted in Fig. 10 and the maximum error is less than $1 \%$.

As for Example II, we also model it using both the thirdorder BCM and FEA methods. The force-displacement relationship can be obtained as shown in Fig. 11. It can be seen that the results obtained by the BCM and FEA match well and the maximum error is $2.5 \%$ as shown in Fig. 12 . The normalized internal axial loads along the beam during the deflection are also displayed in Fig. 12 for comparison. The maximum compressing axial force magnitude $p=21$ is lower than $\left|p_{1}\right|$ and the tip displacement $\Delta Y$ is kept in the intermediate deflection range, so the third-order BCM can correctly capture the force-displacement of the mechanism.

\section{Conclusions}

In this work, we determine the range of the allowable axial force of the third-order BCM. The upper bound $p_{\mathrm{u}}$ are equal to 34.05 , and the lower bound $p_{1}$ is set to different values with respect to different boundary conditions: $p_{1}=-2.47$ for fixed-free buckling, $p_{1}=-20.16$ for fixed-pivoted buckling, and $p_{1}=-39.52$ for fixed-fixed buckling. Generally speaking, a complaint mechanism is analyzed in a series of incremental displacement steps or a series of incremental load

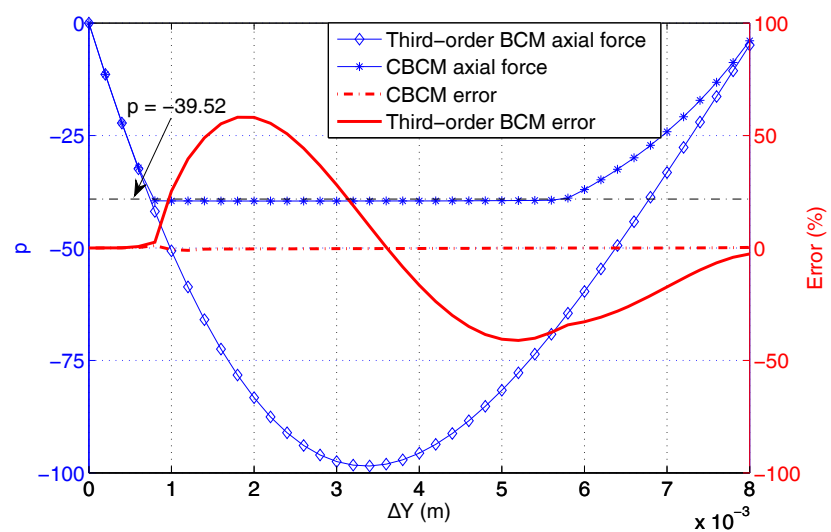

Figure 10. Comparision of normalized axial force and error of Example I.

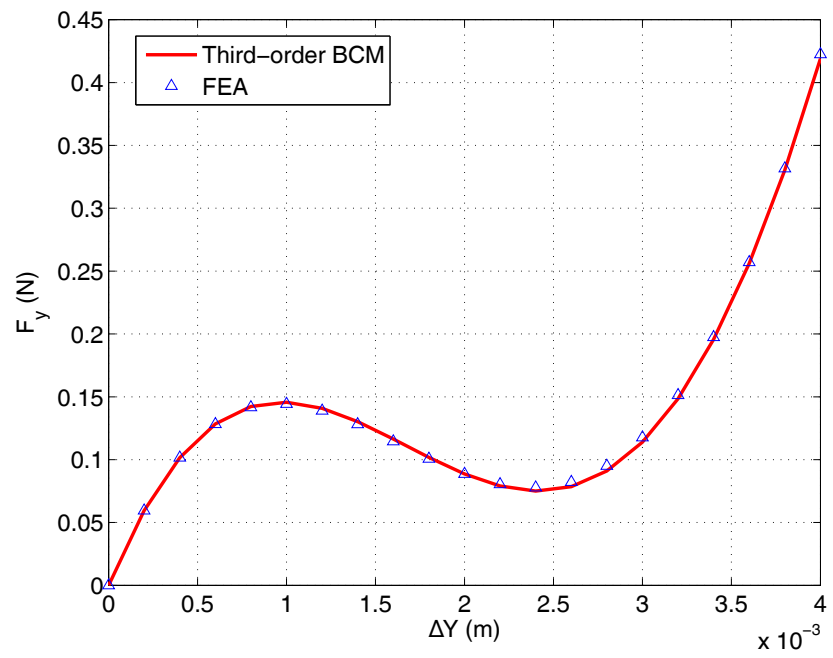

Figure 11. Comparion of the load-displacement relationship of Example II between FEA and the BCM.

steps. At each step, the proposed bounds should be used to check the axial force $p$ to guarantee the effectiveness of the BCM equations.

Three examples are analyzed to demonstrate the effects of the axial force on the modeling errors of the third-order $\mathrm{BCM}$, and the CBCM with 3 elements were used in this work to guarantee the accuracy of the results so that they can be used as the exact solutions for the purpose of comparison. Firstly, the case that the axial tensile force is beyond the upper boundary of the allowable force is given showing the effectiveness of $p_{\mathrm{u}}$. Secondly, the cases that the axial compressing forces arewithin and beyond the lower boundary of the allowable force are presented, the results have verified the effectiveness of the value of $p_{1}$. When using the third-order $\mathrm{BCM}$ for modeling, it is always necessary to check if the axial force is in this allowable range to avoid (in case) large modeling errors. 


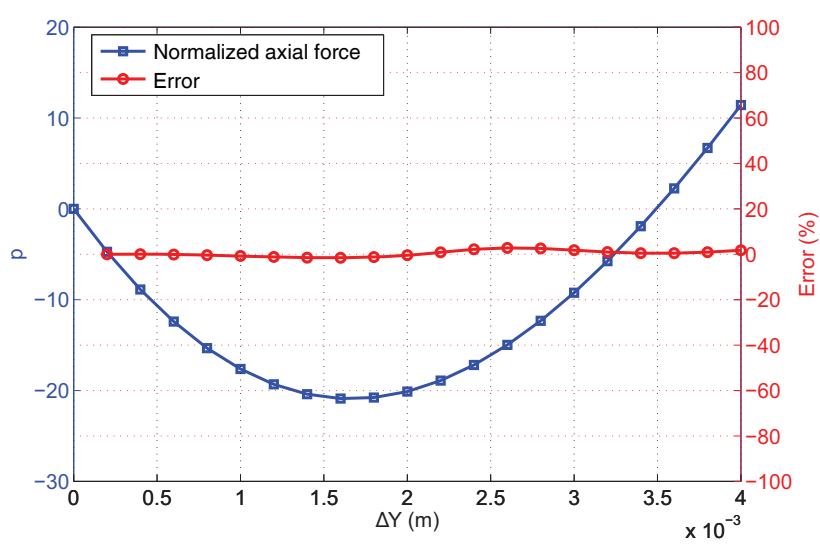

Figure 12. Comparision of normalized axial force and error of Example II.

For the non-dimensional transverse displacements within \pm 0.1 and the normalized axial force $\left(p=P L^{2} /(E I)\right)$ within $\left[p_{1}, p_{\mathrm{u}}\right]$, the majority of the modeling errors of the third-order BCM comes from the truncation of the Taylor's expansion.

It should be acknowledged that the CBCM expands the range of the allowable axial force for the BCM through discretizing the beam (Ma and Chen, 2016). For example, if a beam is discretized into two elements with equal length (the length of each element is $L / 2$ ), then the corresponding normalized compressive axial force of the model for each element becomes $1 / 4$ times of the original normalised one according to Eq. (3). For a beam that carries an axial force beyond the derived bounds in this paper, the chained Beam- Constraint-Model (CBCM) (Ma and Chen, 2016, 2014) and the comprehensive elliptic integral solution (Zhang and Chen, 2013) can be used to model it instead despite they much less analytical than the third-order BCM.

Data availability. All the data used in this manuscript can be obtained on request from the corresponding author.

Competing interests. The authors declare that they have no conflict of interest.

Acknowledgements. The authors gratefully acknowledge the financial support from the National Natural Science Foundation of China under Grant No. 51675396, and the Fundamental Research Funds for the Central Universities under No. JB170403/K5051204021.

Edited by: Xianwen Kong

Reviewed by: two anonymous referees

\section{References}

Awtar, S. and Sen, S.: A Generalized Constraint Model for Two-Dimensional Beam Flexures: Nonlinear LoadDisplacement Formulation, ASME J. Mechan. Des., 132, 081008, https://doi.org/10.1115/1.4002005, 2010a.

Awtar, S. and Sen, S.: A Generalized Constraint Model for Two-Dimensional Beam Flexures: Nonlinear Strain Energy Formulation, ASME J. Mechan. Des., 132, 081009, https://doi.org/10.1115/1.4002006, 2010b.

Awtar, S. and Slocum, A.: Constraint-Based Design of Parallel Kinematic XY Flexure Mechanisms, ASME J. Mech. Des., 129, 816-830, 2007.

Awtar, S., Slocum, A., and Sevincer, E.: Characteristics of BeamBased Flexure Modules, ASME J. Mechan. Des., 129, 625-639, 2007.

Awtar, S., Shimotsu, K., and Sen, S.: Elastic Averaging in Flexure Mechanisms - A Three-Beam Parallelogram Flexure Case Study, ASME J. Mech. Robot., 2, 041006, https://doi.org/10.1115/1.4002204, 2010.

Chen, G. and Bai, R.: Modeling Spatial Deflections of Flexible Beams in Compliant Mechanisms using a Chained Spatial-Beam-Constraint-Model (CSBCM), Proceedings of the ASME Design Engineering Technical Conferences \& Computers and Information in Engineering Conference, Boston, USA, DETC2015-46387, 2-5 August 2015.

Chen, G. and Ma, F.: Kinetostatic modeling of fully compliant bistable mechanisms using Timoshenko beam constraint model, ASME J. Mech. Des., 137, 022301, https://doi.org/10.1115/1.4029024, 2015.

Chen, G., Wilcox, D., and Howell, L.: Fully compliant double tensural tristable micromechanisms (DTTM), J. Micromech. Microeng., 19, 025011, https://doi.org/10.1088/09601317/19/2/025011, 2009.

Gilbert S.: Introduction to Linear Algebra (Fourth Edition), Wellesley Cambridge Press, MA, 2009.

Hao, G.: Extended Nonlinear Analytical Models of Compliant Parallelogram Mechanisms: Third-order Models, T. Can. Soc. Mech. Eng., 39, 71-83, 2015.

Hao, G. and Li, H.: Nonlinear Analytical Modeling and Characteristic Analysis of a Class of Compound Multibeam Parallelogram Mechanisms, ASME J. Mech. Robot., 7, 041016, https://doi.org/10.1115/1.4029556, 2015.

Hao, G., Kong, X., and Reuben, R.: A nonlinear analysis of spatial compliant parallel modules: multi-beam modules, Mech. Mach. Theory, 46, 680-706, 2011.

Holst, G., Teichert, G., and Jensen, B.: Modeling and experiments of buckling modes and deflection of fixed-guided beams in compliant mechanisms, ASME J. Mech. Des., 133, 051002, https://doi.org/10.1115/1.4003922, 2011.

Howell, L. L.: Compliant Mechanisms, Wiley, New York, 2001.

Ma, F. and Chen, G.: Modeling of V-Shape Thermal In-Plane Microactuator Using Chained Beam-Constraint-Model, International Conference on Manipulation, Manufacturing and Measurement on the Nanoscale (3M-NANO 2014), Taipei, p. 296300, 27-31 October 2014.

Ma, F. and Chen, G.: Modeling Large Deflections of Flexible Beams in Compliant Mechanisms using Chained Beam- 
Constraint-Model (CBCM), ASME J. Mech. Robot., 8, 021018, https://doi.org/10.1115/1.4031028, 2016.

Masters, N. and Howell, L.: A self-retracting fully compliant bistable micromechanism, J. Microelectromech. Syst., 12, 273 280, 2003.

Moeen, R. and Moeenfard, H.: A Constraint Model for Beam Flexure Modules with an Intermediate Semi-Rigid Element, Int. J. Mech. Sci., 122, 167-183, 2017.

Moeen, R. and Moeenfard, H.: Load-displacement behavior of fundamental flexure modules interconnected with compliant elements, Mech. Mach. Theory, 120, 120-139, 2018.

Sen, S.: Beam constraint model: generalized nonlinear closed-form modeling of beam flexures for flexure mechanism design, $\mathrm{PhD}$ Dissertation, the University of Michigan, 2013.

Sen, S. and Awtar, S.: A closed-form nonlinear model for the constraint characteristics of symmetric spatial beams, ASME J. Mech. Des., 135, 031003, https://doi.org/10.1115/1.4023157, 2013.
Timoshenko, S.: Strength of Materials: Part I, Elementary Theory and Problems, 2nd ed., Lancaster Press, PA, 2001.

Wilcox, D. and Howell, L.: Fully compliant tensural bistable micromechanisms (FTBM), J. Microelectromech. Syst., 14, 1223 1235, 2005.

Zhang, A. and Chen, G.: A Comprehensive Elliptic Integral Solution to the Large Deflection Problems of Thin Beams in Compliant Mechanisms, ASME J. Mech. Robot., 5, 021006, https://doi.org/10.1115/1.4023558, 2013.

Zhao, H., Bi, S., and Yu, J.: Nonlinear deformation behavior of a beam-based flexural pivot with monolithic arrangement, Precis. Eng., 35, 369-382, 2011. 